\title{
GABA, Not Glutamate, Controls the Activity of Substantia Nigra Reticulata Neurons in Awake, Unrestrained Rats
}

\author{
François Windels and Eugene A. Kiyatkin \\ Behavioral Neuroscience Branch, National Institute on Drug Abuse, Intramural Research Program, National Institutes of Health, Department of Health and \\ Human Services, Baltimore, Maryland 21224
}

\begin{abstract}
Substantia nigra pars reticulata ( $\mathrm{SNr}$ ) receives both GABAergic and glutamatergic (GLU) inputs that are believed to act together to regulate neuronal activity in this structure. To examine the role of these inputs, single-unit recording was coupled with iontophoresis of GLU and GABA in rats under two conditions: awake, unrestrained and under chloral hydrate anesthesia. Although GABA potently inhibited SNr cells in both conditions, freely moving rats showed lower sensitivity than anesthetized animals. Likewise, GLU effectively induced excitations in most SNr neurons in anesthetized animals but was much less effective in awake, unrestrained animals in terms of both the number of sensitive cells and the magnitude of GLU-induced excitation. These findings, along with consistent excitations induced by bicuculline in awake, unrestrained rats, suggest that modulation of GABA inhibitory input, not the opposing actions of GLU and GABA, is the primary factor that regulates the activity state of $\mathrm{SNr}$ neurons.
\end{abstract}

Key words: substantia nigra; GABA; glutamate; bicuculline; single-unit recording; freely moving animals; iontophoresis; anesthesia; basal ganglia

\section{Introduction}

The basal ganglia is a group of subcortical structures implicated in a wide array of functions, such as motor activity, learning, and memory (Alexander et al., 1990; Packard and Knowlton, 2002). Their anatomy and physiology have been studied extensively, and models describing their functional role under normal and pathological conditions have been proposed (Albin et al., 1989; Alexander and Crutcher, 1990; Mink, 1996). According to these models, the striatum receives glutamatergic (GLU) inputs from most cortical areas and thalamus and then sends GABAergic projections to the substantia nigra pars reticulata $(\mathrm{SNr})$ and entopeduncular nucleus (EP) complex through two distinct pathways. The first, or direct pathway, is composed of GABA efferents to the $\mathrm{SNr}$ and EP complex, whereas the second, or indirect pathway, relays successively to the globus pallidus (GP) and then to the subthalamic nucleus. This last structure sends GLU projections to $\mathrm{SNr}$ and EP, which then regulate the activity of specific thalamic nuclei.

On the basis of this structural organization, it is assumed that the activity of SNr cells is regulated primarily by a balance between GABA and GLU inputs. Although the inhibitory and excitatory effects of GABA and GLU on SNr cells have long been known and some of their specific receptor-mediated contributions to the overall neuronal activity have been described (Ham-

\footnotetext{
Received April 22, 2004; revised June 4, 2004; accepted June 8, 2004.

We thank Drs. Roy Wise and Barry Hoffer for valuable comments regarding this manuscript and Leon Brown for editorial assistance.

Correspondence should be addressed to Dr. François Windels, National Institute on Drug Abuse, Intramural Research Program, National Institutes of Health, 5500 Nathan Shock Drive, Baltimore, MD 21224. E-mail: fwindels@intra.nida.nih.gov.

D0I:10.1523/JNEUROSCI.1528-04.2004

Copyright $\odot 2004$ Society for Neuroscience $\quad 0270-6474 / 04 / 246751-04 \$ 15.00 / 0$
}

mond et al., 1978; Collingridge and Davies, 1981; Waszczak and Walters, 1983; Nakanishi et al., 1987; Robledo and Feger, 1990; Celada et al., 1999; Schmitt et al., 1999), most of these studies have been conducted on anesthetized animals. Although this approach provides stable, controlled conditions under which neurons can be studied, the influence of anesthesia as a confounding factor on neural activity and afferent responsiveness remains unclear. This factor may account for some differences noted between studies.

To clarify this issue, we combined single-unit recording with iontophoresis to examine the effects of GLU and GABA on $\mathrm{SNr}$ neurons in rats under awake, unrestrained conditions and under chloral hydrate anesthesia. Therefore, our goals were twofold: to examine the effects of GLU and GABA under physiologically relevant conditions and to reveal the influence of general anesthesia as a factor modulating neuronal activity and afferent responsiveness.

\section{Materials and Methods}

Animals and surgery. Data were obtained from 28 male Long-Evans rats $(400 \pm 50 \mathrm{gm})$ obtained from Charles River Laboratories (Greensboro, $\mathrm{NC})$. All animals were housed individually under standard laboratory conditions (12 hr light/dark cycle beginning at 7:00 A.M.) with food and water available ad libitum. Protocols were performed in compliance with the National Institutes of Health Guide for the Care and Use of Laboratory Animals (Publication 865-23) and were approved by the National Institute on Drug Abuse, Intramural Research Program Animal Care and Use Committee. The surgical procedures used have been described previously (Windels and Kiyatkin, 2003). Briefly, under general anesthesia (equithesin, $0.33 \mathrm{ml} / 100 \mathrm{gm}$, i.p.; dose of sodium pentobarbital, 32.5 $\mathrm{mg} / \mathrm{kg}$ and chloral hydrate, $145 \mathrm{mg} / \mathrm{kg}$ ), rats were implanted with a plastic, cylindrical hub designed to mate with a microelectrode holder (Rebec et al., 1993) during recording. This hub was centered over a hole drilled above the substantia nigra $(5.2-6.0 \mathrm{~mm}$ posterior and $1.4-2.8 \mathrm{~mm}$ lat- 
eral to bregma). After a 3-4 d recovery period, once-daily recording sessions were held over the next $2-5 \mathrm{~d}$ for unanesthetized, unrestrained rats $(n=19)$. A separate group of rats $(n=9)$, prepared as described above, underwent a single recording session under chloral hydrate anesthesia $\left(400 \mathrm{mg} / \mathrm{kg}\right.$, i.p., followed by $\left.120 \mathrm{mg} \cdot \mathrm{kg}^{-1} \cdot \mathrm{hr}^{-1}\right)$. In these experiments, body temperature was monitored and maintained automatically at $37.2 \pm 0.2^{\circ} \mathrm{C}$ with an electric heating pad.

Single-unit recording and iontophoresis. Four-barrel, microfilamentfilled glass pipettes (Omega Dot 50744; Stoelting, Wood Dale, IL), pulled and broken to a diameter of $5 \pm 1 \mu \mathrm{m}$, were used for single-unit recording and iontophoresis. The recording barrel contained 2\% Pontamine Sky Blue in $3 \mathrm{M} \mathrm{NaCl}$, and the balance barrel contained a $0.25 \mathrm{M}$ solution of $\mathrm{NaCl}$. The remaining barrels were filled with solutions of L-GLU monosodium salt $(0.25 \mathrm{M}, \mathrm{pH} 7.5)$, GABA $(0.25 \mathrm{M}$ in $0.125 \mathrm{M} \mathrm{NaCl}, \mathrm{pH} 4)$, or bicuculline methiodide (BIC) $(0.02 \mathrm{~m}$ in $0.125 \mathrm{~m} \mathrm{NaCl}, \mathrm{pH} 4)$. All substances were obtained from Sigma (St. Louis, MO). The resistance of the drug-containing barrels ranged between 10 and $30 \mathrm{M} \Omega$, whereas the recording channel had an impedance of 3-5 $\mathrm{M} \Omega$ (measured at $100 \mathrm{~Hz}$ ). Retaining $( \pm 8-10 \mathrm{nA})$ and ejecting $( \pm 5-60 \mathrm{nA})$ currents were applied with a constant-current generator (Ion 100T; Dagan, Minneapolis, MN). GABA and BIC were applied as cations, and GLU was applied as an anion. Each multibarrel pipette was filled with fresh solution less than $1 \mathrm{hr}$ before use and fixed in a microdrive assembly that later was inserted into the skull-mounted hub. The electrode was then advanced $8.0 \mathrm{~mm}$ below the brain surface to the starting point of unit recording.

Neuronal discharge signals were sent to a head-mounted preamplifier (LF 441CN; National Semiconductor, Santa Clara, CA) and then additionally amplified, filtered (bandpass, $300-3000 \mathrm{~Hz}$ ), and stored on the audio channel of a video cassette recorder. Spike activity was monitored with a digital oscilloscope and audio amplifier and analyzed using a Spike2 interface (Cambridge Electronic Design, Cambridge, UK). After the isolation of single-unit discharges (signal-to-noise ratio of at least 2:1), data collection for each neuron typically lasted 20-30 min. Our protocol typically included several $20 \mathrm{sec}$ applications of GLU, GABA, and BIC performed at $90 \mathrm{sec}$ intervals with different currents $(0$ to \pm 60 $\mathrm{nA})$. All iontophoretic applications used for statistical analysis were performed when the animals were at rest with no sign of overt movements.

Histology. After the last recording session, animals were anesthetized, if not already so, and Pontamine Sky Blue was deposited by current injection ( $-20 \mu \mathrm{A}$ for $20 \mathrm{~min}$ ) at the last recording site. The brain of each rat was then removed and immediately frozen on dry ice. Subsequent histological location of the marked site was made on 25- $\mu$ m-thick frontal sections. The atlas of Paxinos and Watson (1998) served as the basis for histological analyses.

Data analysis. Each iontophoretic test was statistically evaluated, and the response was accepted (i.e., excitation or inhibition) if the mean firing rate during iontophoresis differed significantly $(p<0.05$; two-tail Student's $t$ test) from an equivalent period of baseline activity immediately preceding the iontophoretic application. These responses were also assessed in terms of absolute and relative magnitude, the effect of ejection current (i.e., dose-response relationship), and relationship to the rate of basal activity. Because the duration of each neuronal recording in freely moving rats varied from 5 to $30 \mathrm{~min}$ and the testing program for each unit was different, it was impossible to assess response thresholds and dose-response relationships in each individual unit. Therefore, our data are reported as number of both units and iontophoretic responses. To allow comparisons of GLU and GABA actions between anesthetized and awake, unrestrained conditions, comparable numbers of iontophoretic application of each neurotransmitter were performed in each group. Various relationships between impulse activity and iontophoretic responses were assessed with Student's $t$ tests, Mann-Whitney $U$ tests, correlation, regression analysis, and analysis of covariance (ANCOVA) that were followed by Fisher's post hoc tests.

\section{Results}

Data were obtained from 71 cells histologically verified to be located in SNr; 56 cells were recorded in awake, unrestrained and 25 in anesthetized conditions. In both groups, recorded units were spontaneously active, displaying biphasic spikes of compa-

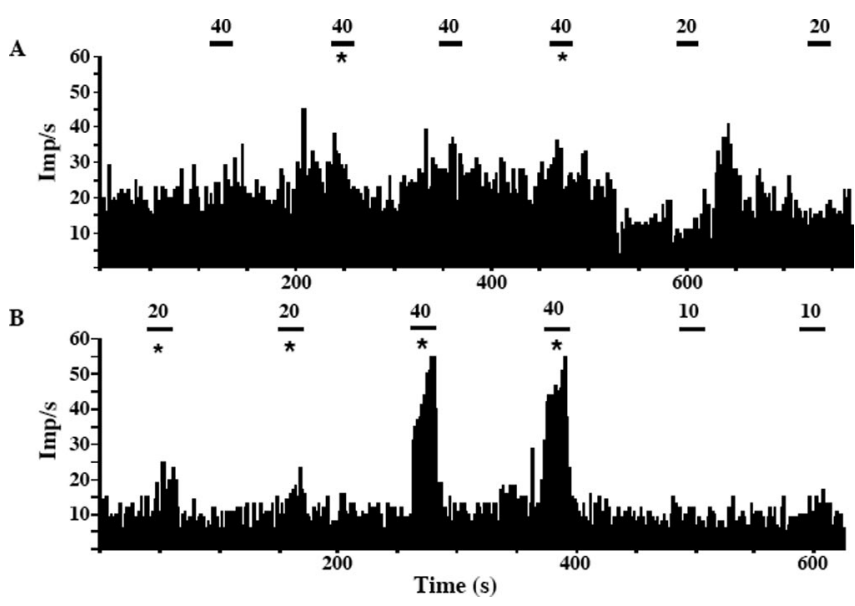

Figure 1. Rate-meter histograms showing individual neuronal responses to iontophoretic GLU applied at different currents in freely moving $(A)$ and chloral hydrate-anesthetized $(B)$ rats. Numbers above each iontophoretic application (solid lines, $20 \mathrm{sec}$ ) indicate ejection currents in nanoamperes. In all cases, neuronal activity is presented as impulses per second (imp/s). Each division of the ordinate represents $5 \mathrm{impulses} / \mathrm{sec}$, and divisions of the abscissa represent $50 \mathrm{sec}$. For each application, asterisks refer to significant ( $p<0.05$; Student's $t$ test) excitation or inhibition.

rable duration (awake, $1.88 \pm 0.25$, range, $1.23-2.23 \mathrm{msec}$; anesthetized, $1.78 \pm 0.16$, range, $1.90-2.19 \mathrm{msec}$ ). In awake, unanesthetized conditions, activity rate assessed before the first iontophoretic application (mean, $28.10 \pm 2.27$; range, $0.60-72.01 \mathrm{impulses} / \mathrm{sec})$ was significantly higher $(p<0.05$; Student's $t$ test) than in anesthetized conditions (mean, $12.68 \pm 1.62$; range, 2.05-37.35 impulses/sec).

\section{GLU responses}

In anesthetized conditions, most $\mathrm{SNr}$ units were highly sensitive to GLU, showing consistent increases in discharge rate in 17 of 20 tested units or in 93 of 109 (87.50\%) GLU applications (Fig. $1 B$ ). The GLU-induced excitation was dose dependent $(p<0.05$; Mann-Whitney $U$ test), with a $315.41 \pm 70.76 \%$ increase at 20 $\mathrm{nA}$ ( 33 increases in 42 tests) and a $535.79 \pm 123.40 \%$ increase at $40 \mathrm{nA}$ ( 42 of 44). In contrast, in freely moving conditions, only 5 of 23 tested units showed consistent GLU-induced excitations after repeated applications. In eight units, no significant changes were observed, whereas 10 other units showed variable responses with weak excitations alternating with weak inhibitions and no changes during repeated GLU applications (Fig. $1 A$ ). The average magnitude of the GLU-induced activation (calculated for 41 of 117 tests with significant increases) was $188.25 \pm 23.58 \%$ at 20 $\mathrm{nA}$ and $151.98 \pm 10.09 \%$ at $40 \mathrm{nA}$. This increase was significantly $(p<0.05$; Mann-Whitney $U$ test) weaker at $40 \mathrm{nA}$ than for the same currents in anesthetized conditions. In both groups, the magnitude of the GLU response was significantly dependent on basal activity rate (anesthetized, $n=93, r=0.51, p<0.001$; freely moving, $n=41, r=0.80, p<0.001$ ). Slower-firing cells showed the largest increase in activity to GLU application.

\section{GABA responses}

In anesthetized conditions, GABA potently affected most $\mathrm{SNr}$ units, showing consistent inhibitions in 17 of 19 tested units or in 97 of $110(88.18 \%)$ applications. At $10-20 \mathrm{nA}$ currents, the firing rate was reduced to $16.60 \pm 2.66 \%$ of the baseline (range, $0.00-$ $86.13 \%)$. Similarly, GABA significantly inhibited the activity of 25 of 27 tested units (115 of 123 tests; 93.49\%) in awake, unrestrained conditions. The average magnitude of the inhibition var- 

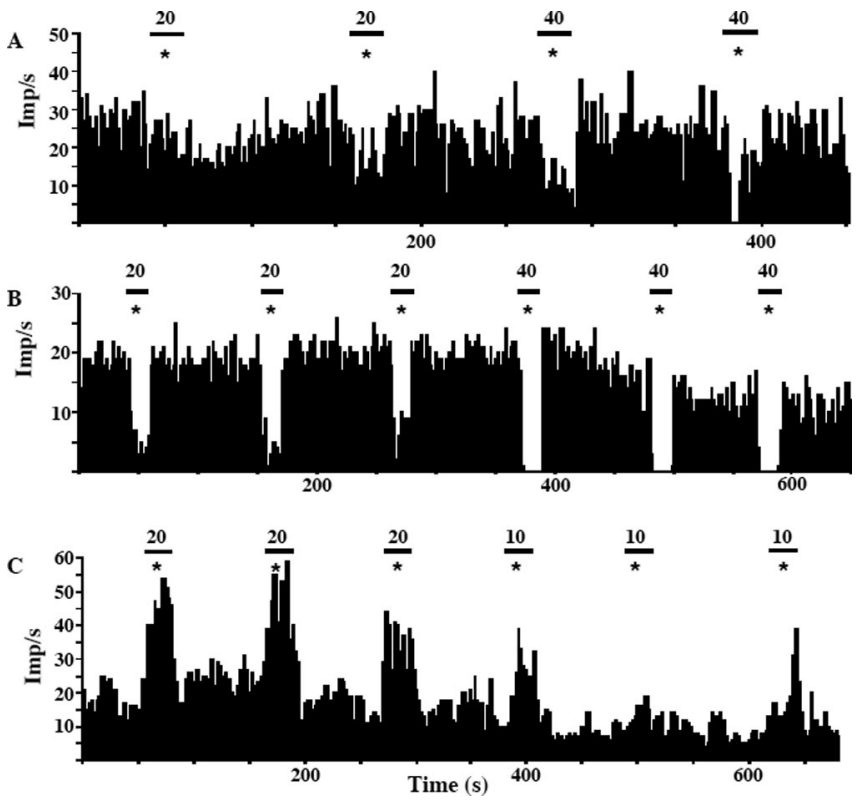

Figure 2. Rate-meter histograms showing individual neuronal responses to iontophoretic GABA and $\mathrm{BIC}$ applied at different currents in freely moving $(A, C)$ and $\mathrm{GABA}$ in chloral hydrateanesthetized $(B)$ rats. Numbers above each iontophoretic application (solid lines, $20 \mathrm{sec}$ ) indicate ejection currents in nanoamperes. In all cases, neuronal activity is presented as impulses per second (imp/s). Each division of the ordinate represents 5 impulses/sec, and divisions of the abscissa represent $50 \mathrm{sec}$. For each application, asterisks refer to significant ( $p<0.05$; Student's $t$ test) excitation or inhibition.

ied between 0.00 and $88.42 \%$ of baseline rate, with a mean of $29.98 \pm 2.74 \%(10-20 \mathrm{nA})$, which was significantly weaker than in anesthetized conditions ( $p<0.001$; Mann-Whitney $U$ test). In both groups, the magnitude of the GABA-induced inhibition in individual units depended on the ejection current (Fig. $2 A, B$ ), as well as on the basal firing rate (anesthetized, $n=97$, $r=0.51, p<0.001$; freely moving, $n=115, r=0.56, p<$ $0.001)$. The GABA-induced inhibition was greater in units with higher discharge rate. The difference observed in the magnitude of the GABA inhibition between the anesthetized and unanesthetized group was independent of the basal firing rate $\left(F_{(1,46)}=4.04 ; p<0.05 ;\right.$ ANCOVA $)$.

\section{$\mathrm{BIC}$ responses}

In freely moving conditions, all $18 \mathrm{SNr}$ units tested were highly sensitive to BIC, showing significant increases of discharge rate in 106 of 122 (94.64\%) tests. In individual units, the BIC-induced excitation was current dependent (Fig. 2C), varying from 110.94 to $792.86 \%$ (mean, $231.54 \pm 13.43 \%$; 5-20 nA) of the baseline. Mean discharge rate during BIC application was $51.26 \pm 3.66$ impulses/sec. When tested, the effect of bicuculline was fully reversed by GABA.

\section{Discussion}

$\mathrm{SNr}$ neurons receive dense GABAergic inputs from the striatum, the GP, and neighboring cells via axon collaterals. This afferent organization gives GABA inhibition a major role in regulating $\mathrm{SNr}$ neuronal activity. Although our study confirms previous work (Waszczak and Walters, 1986; Celada et al., 1999) suggesting that GABA provides a strong inhibitory action on SNr neurons in awake, unrestrained animals, it also reveals that this action is greater in an anesthetized preparation. This difference is probably induced by trichloroethanol, the main metabolite of chloral hydrate and mediator of most of its anesthetic effect. In- deed, Lovinger et al. (1993) demonstrated that trichloroethanol enhances chloride current induced by GABA and thus reinforces the inhibitory action of GABA. This effect may be a factor lowering basal firing rate of the $\mathrm{SNr}$ cells recorded during chloral hydrate anesthesia. Such a mechanism is valid with respect to tonically active GABA afferents from the GP (Smith and Bolam, 1989; Kita and Kitai, 1991), the recurrent projections of SNr cells themselves, and phasically active striatal afferents.

The high GLU sensitivity of SNr units found in anesthetized rats is also strongly supported by abundant neuroanatomical and electrophysiological data (Hammond et al., 1978; Waszczak and Walters, 1983; Robledo and Feger, 1990; Schmitt et al., 1999). Under these conditions, most of the cells tested showed large and consistent increases in firing rate during GLU applications. The number of responsive cells and the magnitude of the effect increased with the dose. In contrast, in awake, unrestrained rats, the effect of GLU was limited in terms of magnitude and number of cells activated. The maximum effect in sensitive units was already reached with the lower current (dose) tested.

The reasons for such unusually low GLU sensitivity of $\mathrm{SNr}$ neurons found in animals under physiologically relevant conditions remain unclear. However, the freely moving condition cannot be the factor responsible for the poor effect of GLU on these cells because, in other structures, we observed dose-dependent and high-magnitude excitations induced by GLU (Kiyatkin and Rebec, 1998, 1999). Because discharge rate is a known factor affecting the magnitude of GLU-induced excitation in striatal (Kiyatkin and Rebec, 1999) and ventral tegmental area neurons (Kiyatkin and Rebec, 1998) and because SNr neurons tested showed the same correlation, higher activity rate in the awake preparation can be a possible factor limiting the GLU response. This factor alone, however, seems unlikely to contribute to a total lack of GLU-induced responses seen in many SNr cells, and it fails to explain the much lower magnitude of GLU-induced excitation in sensitive cells. Previous studies in anesthetized animals with an overall higher basal activity of SNr cells than in the present study, moreover, never reported a lack of GLU-induced excitations (Collingridge and Davies, 1981; Waszczak and Walters, 1983), although it is not clear whether all recorded units were tested with GLU or only the data obtained on GLU-sensitive units were reported. Although we assume that the two sets of experiments were conducted on a comparable population of $\mathrm{SNr}$ cells, anesthesia, through its effect on the GABA inhibitory action discussed above, may have shut down a subclass of cells that are unresponsive to GLU, thus allowing investigation of this population only in unanesthetized rats. Therefore, the population of active $\mathrm{SNr}$ neurons available in awake, freely moving conditions may be different from that in anesthetized conditions.

In contrast to anesthetized conditions in which GLU uniformly excited most SNr neurons, the majority of SNr neurons tested in awake, unrestrained conditions showed variable responses to GLU independent of the current used. This variability, at least in part, may be related to a variable activity state during which units were recorded. Although only the tests performed in quiet, resting conditions with no visually detected overt movements were analyzed, these conditions do not represent a true stationary state. Under these "quiet, resting conditions," environmental factors as well as physiological cycles can affect $\mathrm{SNr}$ cells and, consequently, change their responsiveness to GLU. Several studies revealed that the activity of $\mathrm{SNr}$ neurons is modulated by environmental context (Handel and Glimcher, 2000; Gulley et al., 2002) and by continuous oscillations of the wake-sleep cycle naturally occurring during quiet, resting conditions during day- 
time recording (Miller et al., 1983). Because the activity of serotonin-containing neurons and serotonin release varies depending on the sleep-wakefulness cycle (for review, see Portas et al., 2000) and because this neurotransmitter has direct postsynaptic effects on $\mathrm{SNr}$ neurons via $5-\mathrm{HT}_{2 \mathrm{c}}$ receptors and is involved in presynaptic regulation of GABA release via $5-\mathrm{HT}_{1 \mathrm{~b}}$ receptors (Rick et al., 1995; Stanford and Lacey, 1996), fluctuations in serotonin activity may be a factor determining the variability of basal activity and GLU responsiveness of SNr neurons. Therefore, anesthesia, by completely disrupting the sleep-wakefulness cycle and other cyclic influences and by blocking behavior, may modify the neurophysiology of SNr neurons.

Although in vitro recording of SNr neurons suggests that these cells are autoactive, generating stable high-rate discharges (with rates lower or comparable with those seen in our study) without extrinsic GLU and GABA afferents (Hajos and Greenfield, 1994; Richards et al., 1997), the weak effect of GLU observed in awake, unrestrained animals questions the mechanisms by which $\mathrm{SNr}$ cells can be activated. Because the effect of GABA was predominant on all of the cells tested, modulation of this input (i.e., disinhibition) appears to be the most efficient mechanism for activating $\mathrm{SNr}$ units. Consistent with this mechanism, BIC, a competitive $\mathrm{GABA}_{\mathrm{A}}$ antagonist, induced phasic excitations of all $\mathrm{SNr}$ units tested after low-current brief applications. Not only were all the cells tested sensitive to BIC but the BIC-induced excitations reached a higher mean firing rate than that observed with GLU applications. Interestingly, SNr neurons deprived of extrinsic GLU and GABA input are also activated by BIC (Yuan et al., 2004), suggesting a tonic, GABA-mediated inhibition from axonal collaterals as a factor restraining their impulse activity.

In conclusion, in contrast to the classic hypothesis postulating regulation by direct activation and inhibition, our results suggest that modulation of GABA input may serve as the primary force for both inhibiting and activating SNr units under physiologically relevant conditions. This GABA-mediated, inhibition-disinhibition mode of regulating the activity of SNr neurons might represent, at the neuronal level, the disinhibitory process proposed at the systemic level by Chevalier and Deniau (for review, see Chevalier and Deniau, 1990) for the expression of the basal ganglia function.

\section{References}

Albin RL, Young AB, Penney JB (1989) The functional anatomy of basal ganglia disorders. Trends Neurosci 12:366-375.

Alexander GE, Crutcher MD (1990) Functional architecture of basal ganglia circuits: neural substrates of parallel processing. Trends Neurosci 13:266-271.

Alexander GE, Crutcher MD, DeLong MR (1990) Basal gangliathalamocortical circuits: parallel substrates for motor, oculomotor, "prefrontal" and "limbic" functions. Prog Brain Res 85:119-146.

Celada P, Paladini CA, Tepper JM (1999) GABAergic control of rat substantia nigra dopaminergic neurons: role of globus pallidus and substantia nigra pars reticulata. Neuroscience 89:813-825.

Chevalier G, Deniau JM (1990) Disinhibition as a basic process in the expression of striatal functions. Trends Neurosci 13:277-280.

Collingridge GL, Davies J (1981) The influence of striatal stimulation and putative neurotransmitters on identified neurones in the rat substantia nigra. Brain Res 212:345-359.

Gulley JM, Kosobud AE, Rebec GV (2002) Behavior-related modulation of substantia nigra pars reticulata neurons in rats performing a conditioned reinforcement task. Neuroscience 111:337-349.

Hajos M, Greenfield SA (1994) Synaptic connections between pars compacta and pars reticulata neurones: electrophysiological evidence for functional modules within the substantia nigra. Brain Res 660:216-224.
Hammond C, Deniau JM, Rizk A, Feger J (1978) Electrophysiological demonstration of an excitatory subthalamonigral pathway in the rat. Brain Res 151:235-244.

Handel A, Glimcher PW (2000) Contextual modulation of substantia nigra pars reticulata neurons. J Neurophysiol 83:3042-3048.

Kita H, Kitai ST (1991) Intracellular study of rat globus pallidus neurons: membrane properties and responses to neostriatal, subthalamic and nigral stimulation. Brain Res 564:296-305.

Kiyatkin EA, Rebec GV (1998) Heterogeneity of ventral tegmental area neurons: single-unit recording and iontophoresis in awake, unrestrained rats. Neuroscience 85:1285-1309.

Kiyatkin EA, Rebec GV (1999) Modulation of striatal neuronal activity by glutamate and GABA: iontophoresis in awake, unrestrained rats. Brain Res 822:88-106.

Lovinger DM, Zimmerman SA, Levitin M, Jones MV, Harrison NL (1993) Trichloroethanol potentiates synaptic transmission mediated by gammaaminobutyric acid A receptors in hippocampal neurons. J Pharmacol Exp Ther 264:1097-1103.

Miller JD, Farber J, Gatz P, Roffwarg H, German DC (1983) Activity of mesencephalic dopamine and non-dopamine neurons across stages of sleep and walking in the rat. Brain Res 273:133-141.

Mink JW (1996) The basal ganglia: focused selection and inhibition of competing motor programs. Prog Neurobiol 50:381-425.

Nakanishi H, Kita H, Kitai ST (1987) Intracellular study of rat substantia nigra pars reticulata neurons in an in vitro slice preparation: electrical membrane properties and response characteristics to subthalamic stimulation. Brain Res 437:45-55.

Packard MG, Knowlton BJ (2002) Learning and memory functions of the basal ganglia. Annu Rev Neurosci 25:563-593.

Paxinos G, Watson C (1998) The rat brain in stereotaxic coordinates, Ed 4. San Diego: Academic.

Portas CM, Bjorvatn B, Ursin R (2000) Serotonin and the sleep/wake cycle: special emphasis on microdialysis studies. Prog Neurobiol 60:13-35.

Rebec GV, Langley PE, Pierce RC, Wang Z, Heidenreich BA (1993) A simple micromanipulator for multiple uses in freely moving rats: electrophysiology, voltammetry, and simultaneous intracerebral infusions. J Neurosci Methods 47:53-59.

Richards CD, Shiroyama T, Kitai ST (1997) Electrophysiological and immunocytochemical characterization of GABA and dopamine neurons in the substantia nigra of the rat. Neuroscience 80:545-557.

Rick CE, Stanford IM, Lacey MG (1995) Excitation of rat substantia nigra pars reticulata neurons by 5 -hydroxytryptamine in vitro: evidence for a direct action mediated by 5 -hydroxytryptamine2C receptors. Neuroscience 69:903-913.

Robledo P, Feger J (1990) Excitatory influence of rat subthalamic nucleus to substantia nigra pars reticulata and the pallidal complex: electrophysiological data. Brain Res 518:47-54.

Schmitt P, Souliere F, Dugast C, Chouvet G (1999) Regulation of substantia nigra pars reticulata neuronal activity by excitatory amino acids. Naunyn Schmiedebergs Arch Pharmacol 360:402-412.

Smith Y, Bolam JP (1989) Neurons of the substantia nigra reticulata receive a dense GABA-containing input from the globus pallidus in the rat. Brain Res 493:160-167.

Stanford IM, Lacey MG (1996) Differential actions of serotonin, mediated by $5-\mathrm{HT}_{1 \mathrm{~B}}$ and $5-\mathrm{HT}_{2 \mathrm{C}}$ receptors, on GABA-mediated synaptic input to rat substantia nigra pars reticulata neurons in vitro. J Neurosci 16:7566-7573.

Waszczak BL, Walters JR (1983) Dopamine modulation of the effects of gamma-aminobutyric acid on substantia nigra pars reticulata neurons. Science 220:218-221.

Waszczak BL, Walters JR (1986) Endogenous dopamine can modulate inhibition of substantia nigra pars reticulata neurons elicited by GABA iontophoresis or striatal stimulation. J Neurosci 6:120-126.

Windels F, Kiyatkin EA (2003) Modulatory action of acetylcholine on striatal neurons: microiontophoretic study in awake, unrestrained rats. Eur J Neurosci 17:613-622.

Yuan H, Yamada K, Inagaki N (2004) Multiminute oscillations in mouse substantia nigra pars reticulata neurons in vitro. Neurosci Lett 355: $136-140$. 